\title{
Model fermion Monte Carlo with correlated pairs II
}

\author{
M.H. Kalos \\ Center for Theory and Simulation in Science and Engineering \\ Laboratory of Atomic and Solid State Physics \\ Cornell University \\ Ithaca, New York 14853 \\ K. E. Schmidt \\ Department of Physics and Astronomy \\ Arizona State University \\ Tempe, Arizona 85287
}

\begin{abstract}
Correlated dynamics can produce stable algorithms for excited states of quantum many-body problems. We study a variety of harmonic oscillator problems to demonstrate the kinds of correlations needed. We show that marginally correct dynamics that produce a stable overlap with an antisymmetric trial function give the correct fermion ground state.
\end{abstract}

\section{INTRODUCTION}

We continue the consideration of algorithms that aim at an exact treatment of fermions in the context of quantum Monte Carlo in continuous space. This paper succeeds that of Kalos [1] (MHK) and to some extent Liu, Zhang, and Kalos [2] (LZK), which focussed on pairs of interacting walkers carrying opposite signs or weights. Both papers introduced correlated stochastic dynamics for pairs of random walkers in which the marginal behavior of any one walker was exactly the same as if the partner were absent. The correlations of the members of the pairs, however, avoid the "sign problem." The latter is discussed extensively in both references and elsewhere, so we will not elaborate here, except to say that it causes a decay of signal to noise in fermion Monte Carlo, unless uncontrolled approximations (like imposing a fixed node) are made.

Both LZK and MHK showed how correlated pairs could produce Monte Carlo distributions with stable antisymmetric components so that with the use of a suitable antisymmetric test function, $f_{A}$, exact results could be projected out from which eigenvalues and other physical results could be obtained. LZK showed how purely geometrical correlations could solve an ultra-simplified model problem, the Schrödinger Equation in a parallelogram or in a hypercube. MHK treated another class of model problems, generalized harmonic oscillators in two dimensions, and used correlated walkers in the framework of diffusion Monte Carlo. 
A key idea in the latter paper is that using different correlations depending upon the position and orientation of a pair of antithetic walkers can, in principle break the "plus-minus" symmetry that lies at the heart of the fermion sign problem.

The study of model problems has been extremely fruitful in understanding the role of different dynamics, and of symmetries, especially many-dimensional symmetries. In this paper, we continue the discussion of such models, now including twelve-dimensional (or four-body) harmonic oscillators. In addition to the questions that we have asked of these model problems in the past, we will use them to develop insight about the scaling of the algorithmic complexity with particle number.

It turns out that several of the approaches being considered work particularly well for harmonic oscillators in one or several dimensions. Pairs or other ensembles having particular shapes and orientations are helpful in breaking the "plus-minus" symmetry. The particular form of "drift" implied by the ground state of a harmonic potential preserves such shapes and orientations; we can also arrange to preserve them by appropriate correlations in "diffusion" steps. Thus, creating stable dynamics for a number of states turns out to be easy. This is, of course, a mixed blessing: on one hand one can see immediately the ingredients necessary for stable fermion Monte Carlo in these situations. On the other hand, the task remains of translating the constructions that work for harmonic oscillators into effective algorithms for problems of physical interest.

A conjecture made previously is shown to be correct: if stochastic correlated dynamics gives a stable overlap with an antisymmetric test function, and if the marginal dynamics for any single walker is correct, then that overlap is the same as the one that would be obtained with the lowest antisymmetric wave function. "Correct marginal dynamics" means that integrating out all other members of an ensemble leaves a walker with the dynamics that gives the ground state (except for possible cancellation of plus and minus walkers.)

We will demonstrate the exact Green's function for a finite time step in diffusion Monte Carlo in which the importance function is the symmetric solution of a many-dimensional harmonic oscillator. Dynamics for pairs or larger ensembles of walkers with various correlations can be constructed from that Green's function whose marginal dynamics for one walker is exact.

Using this result, we can exhibit the asymptotic behavior in large imaginary time of correlated walks using both "parallel dynamics" and "reflected dynamics with cancellation," in the language of MHK, and show that the distributions give the exact antisymmetric wavefunctions.

The transformation that maps the solution of the Schrödinger equations with pair-wise harmonic oscillator potentials and with central harmonic oscillator potentials is given explicitly. It provides a motivation for solving problems of the latter kind that describe three- and four-body fully spin-polarized systems. The constructions are easiest when the correlated ensembles are respectively quartets (on hyperrectangles) and octets (on hypercubes) but we show how correlated pairs can be used throughout. This will provide some insight on complexity scaling. 


\section{CORRELATED DIFFUSION OF ENSEMBLES OF SIGNED WALKERS}

Green's function and diffusion Monte Carlo deal with ensembles of walkers $\left\{R_{k}\right\}$ that are usually treated as independent, except for the correlations that arise from branching processes and possibly from population control.

In proposing to deal with antisymmetric solutions of continuum problems, we introduce ensembles of signed walkers, $\left\{R_{k}^{+}, R_{k}^{-}\right\}$that permit the representation of wave functions that are not everywhere positive. Thus (without any importance sampling) we represent the antisymmetric wave function as

$$
\psi_{A}(R)=\sum_{k}\left[\delta\left(R-R_{k}^{+}\right)-\delta\left(R-R_{k}^{-}\right)\right]
$$

We may calculate the overlap with some antisymmetric test function $f_{A}(R)$ as

$$
\int f_{A}(R) \psi_{A}(R) d R=\sum_{k}\left[f_{A}\left(R_{k}^{+}\right)-f_{A}\left(R_{k}^{-}\right)\right]
$$

A stable and efficient method must produce ensembles in which walkers with positive weights $R_{k}^{+}$remain mostly in regions of configuration space in which $\psi_{A}>0$ and $R_{k}^{-}$stay mostly where $\psi_{A}<0$. Imposing the fixed-node constraint on the walkers has an analogous effectnamely that $R_{k}^{+}$exist only where $\psi_{A T}(R)>0$ for some trial antisymmetric function.

Unfortunately, most stochastic dynamics, especially those that treat walkers as independent have symmetric solutions as their asymptotic distributions- those for which the overlaps computed by Eq. 2.2 decay exponentially to zero. More generally, if the algorithm obeys the "plus-minus symmetry" in which an interchange of all of the plus and minus labels on the walkers leaves the dynamical rules unchanged, then there will be an exponential decay of the overlap signal (and an exponential decay of signal to noise.)

Thus one must consider correlated dynamics. The simplest ensembles to use are pairs of plus and minus walkers. We will give some attention to the question of whether one needs larger ensembles for the model problems discussed here.

MHK pointed out that various classes of correlations are very easy to introduce with the framework of importance-sampled diffusion Monte Carlo. We repeat some of the exposition that was given there.

Importance sampling seeks to generate ensembles of walkers that have the distribution

$$
\psi_{T}(R) \psi(R)
$$

rather than simply $\psi(R)$, for some $\psi_{T}(R)$.

Ceperley [3] derived a general approximate set of dynamical rules to carry this out. For a finite time interval $\delta \tau$ a walker at $R$ is moved according to the following three steps:

Drift: a walkers is moved to $R_{d}$ with

$$
R_{d}=R+\delta \tau \frac{\vec{\nabla} \psi_{T}(R)}{\psi_{T}(R)}
$$

Diffusion: a walker is moved to $R^{\prime}$ with 


$$
R^{\prime}=R_{d}+\sigma \vec{U}
$$

where for an $N$-body system, $\vec{U}$ is a $3 N$-dimensional vector, each of whose components is an independent normal random variable with mean zero and variance one. Appropriate values of $\sigma$ are discussed below.

Branching: each walker becomes $M$ walkers where $\langle M\rangle$, the expected value of $M$, is

$$
\langle M\rangle=\exp \left\{-\delta \tau\left[H \psi_{T} / \psi_{T}-E_{T}\right]\right\}
$$

where $H$ is the hamiltonian of the system, and $E_{T}$ is some trial eigenvalue. An alternative to branching, which we will use later in our analysis, is to attach a weight $W$ to each walkers so that the last step is replaced by:

$$
W \rightarrow W \exp \left\{-\delta \tau\left[H \psi_{T} / \psi_{T}-E_{T}\right]\right\}
$$

In Ceperley's approximate treatment, $\sigma$ is given by

$$
\sigma^{2}=\delta \tau
$$

In most applications of these ideas to antisymmetric states, $\psi_{T}(R)$ is itself taken to be antisymmetric. Here, by contrast, we take it to be the symmetric ground state:

$$
\psi_{T}(R)=\psi_{0}(R)=\exp \left(-R^{2} / 2\right)
$$

The motivation (cf. MHK) is that it leads to dynamics in which pairs (or other ensembles) are preserved. This will be particularly striking for the harmonic oscillator model systems considered here.

When the form of Eq. 2.6 is used for $\psi_{T}(R)$, then Eq. 2.4a describing the drift becomes:

$$
R_{d}=(1-\delta \tau) R
$$

We show in the appendix that an exact finite time Green's function for diffusion Monte Carlo importance sampled with the harmonic oscillator ground state can be written. It has the same structure as Eqs. 2.4 and 2.7 except that the drift term is now

$$
R_{d}=e^{-\delta \tau} R \equiv \rho R
$$

and

$$
\sigma^{2}=\left(1-e^{-2 \delta \tau}\right) / 2 \equiv\left(1-\rho^{2}\right) / 2
$$

Finally, when $\psi_{T}(R)=\psi_{0}(R), H \psi_{T} / \psi_{T}=N / 2$, so that the branching factor or weight multiplication factor is a constant. We will take $E_{T}$ to be the excited state eigenvalue.

Eq. 2.8a prescribes simply a rescaling of all coordinates by a constant factor: that means that geometrical shapes and orientations of correlated ensembles are preserved in drift steps. Pairs remain pairs with the same direction. Parallel pairs remain parallel pairs. Drift preserves ensembles of parallel squares as such.

The fact that $\sigma^{2}$ is a constant permits us to relate the vectors $\vec{U}$ in simple geometric ways in a correlated ensemble. Thus, for a pair $\left\{R_{d k}^{+}, R_{d k}^{-}\right\}$after drift, we may set 


$$
\vec{U}_{k}^{-}=\vec{U}_{k}^{+}
$$

to get "parallel dynamics", in which again geometrical shapes and orientations are preserved. For a pair, an alternative to Eq. 2.9 is "reflected dynamics" defined in the following way:

Let

$$
\begin{gathered}
\vec{\Omega}_{k}=\frac{R_{d k}^{+}-R_{d k}^{-}}{\left|R_{d k}^{+}-R_{d k}^{-}\right|} \\
\vec{U}_{k}^{-}=\vec{U}_{k}^{+}-2\left(\vec{U}_{k}^{+} \cdot \vec{\Omega}_{k}\right) \vec{\Omega}_{k}
\end{gathered}
$$

This transformation produces isotropic random vectors $\vec{U}_{k}^{-}$with the property that $\left|\vec{U}_{k}^{-}\right|^{2}=$ $\left|\vec{U}_{k}^{+}\right|^{2}$; they are reflected in the figure that bisects the line from $R_{d k}^{-}$to $R_{d k}^{+}$.

Another important kind of dynamics uses the correlation of Eq. 2.10 but carries out cancellation based on the subtraction of the gaussian kernels that describe the diffusion for a pair of opposite walkers.

$$
G_{\text {pair }}(R)=\left\{\exp \left[-\left(R-R_{d}^{+}\right)^{2} /\left(2 \sigma^{2}\right)\right]-\exp \left[-\left(R-R_{d}^{-}\right)^{2} /\left(2 \sigma^{2}\right)\right]\right\} /\left(2 \pi \sigma^{2}\right)^{3 N / 2}
$$

Where $G_{\text {pair }}(R)>0$, new points $R^{+}$may appear; when $G_{\text {pair }}(R)<0$, they do not. Similarly, new $R^{-}$appear only where $G_{\text {pair }}(R)<0$. Note also that $G_{\text {pair }}(R)$ maps into its negative on reflection in the line (or plane or hyperplane) that is the perpendicular bisector of the line from $R_{d}^{+}$to $R_{d}^{-}$. New points $R^{+}$and $R^{-}$may be taken as reflections of each other, preserving the orientation of pairs. We call this "Reflection Dynamics with Cancellation," or simply "RC" dynamics. Generalizations will be given below, as needed for specific states.

Creating the necessary correlations within an ensemble by simple transformations of $\vec{U}$, as we have done, ensures that the collective dynamics of the ensemble is marginally correct: each walker by itself is subject to a diffusion step drawn from a correct gaussian, as if no other walkers were present. Cancellation implicit in the use of $G_{\text {pair }}(R)$ does not change the average behavior of the density that follows from the descendents of that walker.

One more introductory technical comment concerns the generalization of Eq. 2.2 when importance sampling with $\psi_{0}(R)$ is used. The general form of an overlap integral becomes:

$$
\begin{gathered}
\int f_{A}(R) \psi_{A}(R) d R=\int\left[\frac{f_{A}(R)}{\psi_{0}(R)}\right] \psi_{0}(R) \psi_{A}(R) d R \\
=\sum_{k}\left[\frac{f_{A}\left(R_{k}^{+}\right)}{\psi_{0}\left(R_{k}^{+}\right)}-\frac{f_{A}\left(R_{k}^{-}\right)}{\psi_{0}\left(R_{k}^{-}\right)}\right]
\end{gathered}
$$

\section{STABLE CORRECT ALGORITHMS}

It is necessary that any correlations between walkers introduced in the interests of stability not modify the desired solution of the Schrödinger equation. 
A transient estimate calculation [4] begins by sampling positive and negative walkers from the positive and negative parts of a fermion trial wave function. Both populations are then propagated using the same dynamics as in the usual Bose ground-state calculation. Expectation values require computing the overlap of the resulting population with an antisymmetric trial function, usually the same as the original trial function. As is well known, the result is unstable in the sense that the overlap and the signal-to-noise ratio decay exponentially to zero. However, since the correct Green's function is used, the results are correct on the average if the signal can be extracted at long enough times.

Thus, in transient estimation, any single walker obeys the correct dynamics. To make a stable algorithm, we need to modify the dynamics without destroying the correctness of the results. That is to say, the overlap integrals must have the same Monte Carlo expectation values as given by the original dynamics. One modification is to cancel walkers. If a pair of walkers, one positive and one negative, are at exactly the same point, their contribution to all expectation values cancel at all future times. The pair can therefor be dropped from the calculation without changing the average values of any overlap integrals. This means further that pairs of walkers can be omitted with the probability that they move to the same point. Arnow et al. [5] exploited this simple cancellation idea to solve some model few-body problems; however, cancellation alone does not break the plus-minus symmetry and does not produce algorithms that scale well with the number of particles. Exponentially large populations are required as the number of particles increases.

A second ingredient is correlated dynamics. Ignoring cancellation for a moment, we get correct answers if, when we view any single walker alone, its dynamics are unchanged. The computation of overlap integrals is linear in the density of random walkers; correlations between walkers do not change the Monte Carlo expectations. In the examples of section II, walker pairs make correlated moves since the diffusion steps use correlated gaussian variables, but each walker seen alone executes the same walk that it would in the absence of the other, and therefor gives correct overlaps. We call such dynamics marginally correct. More generally, any expectation value calculated with an ensemble of walkers with marginally correct dynamics will be correct.

Thus, the inclusion of cancellation in the correlated dynamics also yields a correct algorithm; the expectations of overlap integrals remain unchanged. If we combine correlated dynamics with cancellation, we can develop stable algorithms. When the correlated dynamics are marginally correct and give a stable overlap with an antisymmetric trial function, the signal to noise ratio does not decay, and the results must be correct.

\section{ONE-DIMENSIONAL HARMONIC OSCILLATOR}

It is instructive to analyze completely the dynamics of pairs for the one-dimensional harmonic oscillator, Consider first the case of parallel dynamics for a pair, $\left\{x_{0}^{+}, x_{0}^{-}\right\}$. We assume a series of time steps with constant $\delta \tau$. Drift steps transform $x$ into $e^{-\delta \tau} x \equiv \rho x$. Parallel dynamics mean that positions of both the plus and minus walkers after drift are incremented by the same gaussian $u$, with mean zero and variance $\left(1-\rho^{2}\right) / 2$. Then

$$
x_{0}^{+} \rightarrow \rho x_{0}^{+}+u_{1} \rightarrow \rho\left[\rho x_{0}^{+}+u_{1}\right]+u_{2} \ldots \rightarrow \rho^{k} x_{0}^{+}+\sum_{l=1}^{k} \rho^{k-l} u_{l} \rightarrow \ldots \rightarrow \lim _{k \rightarrow \infty} \sum_{n=0}^{k-1} \rho^{n} u_{k-n}
$$




$$
x_{0}^{-} \rightarrow \rho x_{0}^{-}+u_{1} \rightarrow \rho\left[\rho x_{0}^{-}+u_{1}\right]+u_{2} \ldots \rightarrow \rho^{k} x_{0}^{-}+\sum_{l=1}^{k} \rho^{k-l} u_{l} \rightarrow \ldots \rightarrow \lim _{k \rightarrow \infty} \sum_{n=0}^{k-1} \rho^{n} u_{k-n}
$$

Thus $\delta x_{k}$, the relative distance between $x_{k}^{+}$and $x_{k}^{-}$decreases by a factor $\rho$ after each drift step:

$$
\delta x_{0}=x_{0}^{+}-x_{0}^{-} \rightarrow e^{-\delta \tau} \delta x_{0} \rightarrow e^{-2 \delta \tau} \delta x_{0} \rightarrow e^{-3 \delta \tau} \delta x_{0} \rightarrow \ldots e^{-\tau} \delta x_{0}
$$

after total imaginary time $\tau$. Finally, we attach a weight to each pair; that weight is multiplied by the factor $e^{\delta \tau}$ instead of branching. The latter factor takes account of the difference between the eigenvalue of the first excited state $(3 / 2)$ and the ground state $(1 / 2)$ from which the "local energy," $H \psi_{0} / \psi_{0}$, is computed. Thus an initial weight $W_{0}$ is transformed successively into:

$$
W_{0} \rightarrow e^{\delta \tau} W_{0} \rightarrow e^{2 \delta \tau} W_{0} \rightarrow e^{3 \delta \tau} W_{0} \rightarrow \ldots e^{\tau} W_{0}
$$

The product $\delta x_{k} W_{k}$ is constant, equal to $\delta x_{0} W_{0}$.

As $\delta x_{k} \rightarrow 0$, the pair reduces to a point whose position has the distribution $\psi_{0}^{2}(x)$, since by construction the marginal dynamics for either point $\left\{x_{k}^{+}, x_{k}^{-}\right\}$is that which gives the ground state $\psi_{0}(x)$ modified by the importance function $\psi_{0}(x)$, i.e., $\exp \left(-x^{2}\right)$. This result can also be seen by inspection of Eqs. 4.1a and 4.1b. They show explicitly that $x_{k}^{+} \rightarrow x_{k}^{-}$. Each is seen as a sum of gaussian random variables weighted with $\rho^{n}$. The sums, i.e. the values of $x_{k}^{ \pm}$, are then also gaussian random variables with mean zero. The variance of the limiting distribution is $\left(1-\rho^{2}\right) / 2 \cdot \sum_{1}^{\infty} \rho^{2 n}=1 / 2$. That is, the distribution is again $\exp \left(-x^{2}\right)$.

The overlap of any antisymmetric function $f_{A}$ with the solution generated by the pairs is therefor:

$$
\begin{gathered}
\int f_{A}(x) \psi_{A}(x) d x=\int \frac{f_{A}(x)}{\psi_{0}(x)} \psi_{0}(x) \psi_{A}(x) d x \\
=\sum_{k} W_{k}\left[\frac{f_{A}\left(x_{k}^{+}\right)}{\psi_{0}\left(x_{k}^{+}\right)}-\frac{f_{A}\left(x_{k}^{-}\right)}{\psi_{0}\left(x_{k}^{-}\right)}\right] \\
\rightarrow \sum_{k} W_{k} \delta x_{k} \frac{d}{d x}\left[\frac{f_{A}(x)}{\psi_{0}(x)}\right] \\
=\delta x_{0} W_{0} \int\left\{\frac{d}{d x}\left[\frac{f_{A}(x)}{\psi_{0}(x)}\right]\right\} \psi_{0}^{2}(x) d x \\
=-2 \delta x_{0} W_{0} \int\left[\frac{f_{A}(x)}{\psi_{0}(x)}\right] \psi_{0}(x) \frac{d}{d x} \psi_{0}(x) d x=2 x_{0} W_{0} \int f_{A}(x) x e^{-x^{2} / 2} d x
\end{gathered}
$$

exactly the right answer.

Thus, for this simple problem, parallel dynamics is asymptotically stable and exact. 
When "reflected dynamics with cancellation" (RC) is applied to this problem, the only change is that at the "diffusion" step, equal and opposite gaussian increments are added to $x^{+}$and $x^{-}$, respectively. The sum $X_{k}=x_{k}^{+}+x_{k}^{-}$is unchanged by the diffusion, but is decreased by $e^{-\delta \tau}$ at every drift. Since $x_{k}^{+}>x_{k}^{-}$remains true throughout, $x_{k}^{+} \approx \exp (-k \delta \tau)$, and

$$
x_{k}^{-} \rightarrow-x_{k}^{+}
$$

The equilibrium distribution generated by these rules satisfies the following equation:

$$
\begin{gathered}
\chi(x)=e^{\delta \tau} \int \frac{e^{-[x-\rho z]^{2} /\left(2 \sigma^{2}\right)}-e^{-[x+\rho z]^{2} /\left(2 \sigma^{2}\right)}}{\sqrt{2 \pi \sigma^{2}}} \chi(z) d z \\
\sigma^{2}=[1-\exp (-2 \delta \tau)] / 2=\left(1-\rho^{2}\right) / 2
\end{gathered}
$$

The fact that the "source" positions in the two gaussians are negatives of each other follow from the asymptotic behavior indicated by Eq. 4.8 above (or equally for all steps if the initial values satisfied $x_{0}^{-}=-x_{0}^{+}$.)

The factor $e^{\delta \tau}$ in front reflects the growth in population or weight from branching. The factor $\rho$ in the exponents of the gaussian terms reflects the shift of the source positions because of the drift.

The expression

$$
\chi(x)=x e^{-x^{2}}
$$

is a solution of Eq. 4.9, and is again, the exact (first) antisymmetric wave function for a harmonic oscillator in one dimension, with an extra factor $\psi_{0}(x)$ from importance sampling.

We note also that one can mix the dynamics, using parallel dynamics with probability $p_{\|}$and RC dynamics with probability $1-p_{\|}$. This combination works for any value of $p_{\|}$.

Of course, finding antisymmetric solutions in one dimension, even by naive Monte Carlo methods, is no challenge: here as always, the nodal point is at $x=0$, and that fact can be used as a boundary condition to get the exact solution. It is important to observe, however, that for parallel or for mixed dynamics, the walkers do not respect the nodal point restriction, so that we have a generalization of the fixed-node condition.

\section{TWO-DIMENSIONAL HARMONIC OSCILLATOR}

The generalization of the methods of the previous section to find antisymmetric solutions of the form

$$
\psi_{01}(x, y)=y e^{-\left(x^{2}+y^{2}\right)}
$$

is immediate and uninteresting. This was already discussed in MHK. One simply uses pairs of walkers, $\left\{\vec{r}^{+}, \vec{r}^{-}\right\}$with

$$
x^{+}=x^{-} ; y^{+}>y^{-}
$$


Parallel, RC, and mixed dynamics, all preserve these conditions, and the distributions in $x$ and $y$ are independent; the distribution in $x$ is that of the ground state in one dimension, and that in $y$ is that of the corresponding first excited state.

On the other hand, if we seek the solution

$$
\psi_{11}(x, y)=x y e^{-\left(x^{2}+y^{2}\right) / 2}
$$

something new is needed.

Following the development of Eqs. 4.3-4.7, we have

$$
x y e^{-\left(x^{2}+y^{2}\right) / 2}=\frac{\partial^{2}}{\partial x \partial y} e^{-\left(x^{2}+y^{2}\right) / 2} .
$$

We surmise, therefor, that a quartet of walkers, $\left\{{\overrightarrow{r_{1}}}^{+},{\overrightarrow{r_{1}}}^{-},{\overrightarrow{r_{2}}}^{+},{\overrightarrow{r_{2}}}^{-}\right\}$with

$$
\begin{aligned}
& x_{1}^{+}=x_{1}^{-} ; y_{1}^{-}<y_{1}^{+} \\
& x_{2}^{+}<x_{1}^{-} ; y_{2}^{+}=y_{1}^{-} \\
& x_{2}^{-}=x_{2}^{+} ; y_{2}^{-}>y_{2}^{+} \\
& x_{1}^{+}>x_{2}^{-} ; y_{1}^{+}=y_{2}^{-}
\end{aligned}
$$

will be stable and give the correct solution for parallel dynamics (in which all four walkers are given the same two-dimensional gaussian increment.)

Both drift and parallel diffusion transform a rectangle into another rectangle with the same orientation: drift determined by the ground state of a harmonic oscillator shrinks a rectangle into a smaller one, and diffusion in which all walkers move with the same gaussian vector simply displaces the drifted rectangle. Furthermore, the shrinking of those rectangles to a point goes on as in one dimension, so that the replacement of the sums and differences of estimators by derivatives is the same as in one dimension. Hence, the integration by parts leads once more to the correct excited state.

Reflected dynamics may be introduced much as in one dimension, provided we reflect in the $y$ and $x$ axes in turn. That is, if $\left\{u_{x}, u_{y}\right\}$ are two gaussian random variables with mean zero and variance $\left(1-\rho^{2}\right) / 2$, then one increments the positions of the four walkers after drift in the following pattern:

$$
\begin{aligned}
& x_{1}^{+}=x_{d 1}^{+}+u_{x} ; y_{1}^{+}=y_{d 1}^{+}+u_{y} \\
& x_{1}^{-}=x_{d 1}^{-}+u_{x} ; y_{1}^{-}=y_{d 1}^{-}-u_{y} \\
& x_{2}^{+}=x_{d 2}^{+}-u_{x} ; y_{2}^{+}=y_{d 2}^{+}-u_{y} \\
& x_{2}^{-}=x_{d 2}^{-}-u_{x} ; y_{2}^{-}=y_{d 2}^{-}+u_{y}
\end{aligned}
$$


These correlated diffusions also transform a rectangle into another with the same orientation. Finally, we remark that when the gaussian kernels that describe the diffusion are added up with appropriate signs (the obvious generalization of Eq. 2.11) as is appropriate for cancellation, the composite kernel is positive or negative in quadrants defined by the center of the rectangle of source positions, and simply changes sign on reflection in coordinate axes that pass through that center. Hence we may correctly select correlated post-diffusion coordinates for the four walkers that also lie on a rectangle with the same orientation. We expect, therefor, that reflected dynamics with cancellation will also be stable and accurate, and this is indeed the case.

It appears that this next excited state requires a quartet of walkers for stability. That is not the the case. We consider pairs that are oriented either vertically:

$$
\begin{aligned}
& x^{+}=x^{-}>0 ; \quad y^{+}=-y^{-}>0 \\
& x^{+}=x^{-}<0 ; \quad y^{+}=-y^{-}<0
\end{aligned}
$$

or horizontally:

$$
\begin{aligned}
& x^{+}=-x^{-}>0 ; \quad y^{+}=y^{-}>0 \\
& x^{+}=-x^{-}<0 ; \quad y^{+}=y^{-}<0 .
\end{aligned}
$$

These are symmetric about either the $x$ or $y$ axes. One can switch between one and the other by reflecting a walker in the origin. Consider a vertical pair as in Eq. 5.7a. RC dynamics preserves it as a vertical symmetric pair. Suppose also that $x^{+}>y^{+}$, and that we follow it as long as this inequality holds. When, however, $x^{+}<y^{+}$for the first time, transform the pair:

$$
\left\{\left(x^{+}, y^{+}\right),\left(x^{+},-y^{+}\right)\right\}
$$

into the equivalent horizontal pair

$$
\left\{\left(x^{+}, y^{+}\right),\left(-x^{+}, y^{+}\right)\right\}
$$

and follow it by $\mathrm{RC}$ dynamics as long as $x^{+}<y^{+}$, after which it becomes a vertical pair again.

This modified dynamics, involving pairs only, is stable.

The process of waiting until a pair crosses the pair of lines

$$
x^{2}=y^{2}
$$

can be delicate when $\delta \tau$ is not extremely small (since the random walk can also cross the coordinate axes at the same time.) In principle, this problem can be avoided by causing a diffusion process exactly to the boundaries of Eq. 5.10, by generating a "first passage" event (as described, for example in LZK, section IV). The outcome is an exact dynamics (no time step error) that is stable as well. 
A contrasting situation arises when we consider the state whose nodal line is a single circle:

$$
\psi_{20}(\vec{r})=\left(1-r^{2}\right) e^{-r^{2} / 2}
$$

A function not orthogonal to this is generated by differentiating twice with respect to either $x$ or $y$. This suggests that four walkers on a line is a suitable ensemble to generate solutions like that of Eq. 5.10. The signs are distributed differently, namely, as,,,-++proceeding along the line. A suitable initial arrangement of the walkers would be on the $x$ axis, at positions:

$$
x_{1}^{-}<x_{1}^{+}<0<x_{2}^{+}=-x_{1}^{+}<x_{2}^{-}=-x_{1}^{-}
$$

As before, drift determined by the harmonic oscillator ground state preserves the collinearity of ensembles of walkers. Parallel dynamics does the same, as does reflected dynamics with cancellation in which the two plus-minus pairs are both reflected in their perpendicular bisectors.

It is possible to carry this out as well using two walkers rather than four. To do that, it is necessary again to use RC dynamics, supplemented with a boundary condition that the remaining plus walker be reflected on a line passing through the origin and perpendicular to the line joining the walkers.

\section{NINE-DIMENSIONAL HARMONIC OSCILLATOR}

The last and most complex harmonic oscillator state that we treat in this paper is that of three particles bound to a center of force by harmonic potentials:

$$
H=\left[-\nabla_{1}^{2}-\nabla_{2}^{2}-\nabla_{3}^{2}+r_{1}^{2}+r_{2}^{2}+r_{3}^{2}\right] / 2
$$

This is equivalent to the problem of three particles with pairwise springs, when the origin is taken to be the center of mass, as can be seen from the identities

$$
\begin{aligned}
\sum_{i<j}\left|\vec{r}_{i}-\vec{r}_{j}\right|^{2} & =\frac{1}{2} \sum_{i, j}^{N}\left|\vec{r}_{i}-\vec{r}_{j}\right|^{2} \\
& =N \sum_{i}^{N}\left(r_{i}^{2}-R_{c m}^{2}\right) \\
& =N \sum_{i}^{N}\left|\vec{r}_{i}-\vec{R}_{c m}\right|^{2}
\end{aligned}
$$

where $\mathrm{N}$ is the number of particles and $\vec{R}_{c m}$ is the center of mass position.

We now attach spins of one half to each particle. The state in which two spins are equal requires pairs of walkers starting with the orientation:

$$
\begin{array}{cccccccccc}
\text { plus } & x_{1} & y_{1} & z_{1} & x_{2} & y_{2} & z_{2} & x_{3} & y_{3} & z_{3} \\
\text { minus } & x_{1} & -y_{1} & z_{1} & x_{2} & y_{2} & z_{2} & x_{3} & y_{3} & z_{3}
\end{array}
$$


The application of either parallel dynamics or reflected dynamics with cancellation preserves the identity and orientation of the pairs, and will therefor give a stable overlap with a test function that contains the p-orbital $y \exp \left(-R^{2} / 2\right)$. This is the same construction as has been considered above, except for the additional eight dimensions.

We consider instead the state obtained by attaching equal spins of one half to each particle in a fully spin-polarized state. A Slater determinant of ground state and p-wave orbitals may be formed:

$$
\psi_{A}(R)=\left[x_{1}\left(y_{2}-y_{3}\right)+x_{2}\left(y_{3}-y_{1}\right)+x_{3}\left(y_{1}-y_{2}\right)\right] \exp \left(-R^{2} / 2\right)
$$

Any of the terms in this function can be obtained from the ground state by differentiating with respect to some $x_{i}$ and $y_{j}, i \neq j$. We may use $x_{1}$ and $y_{2}$, for example. Then introduce four walkers (respectively plus then minus cyclically around the figure) arranged initially in a hyperrectangle as follows:

$\begin{array}{cccccccccc}\text { plus } & x_{1} & y_{1} & z_{1} & x_{2} & y_{2} & z_{2} & x_{3} & y_{3} & z_{3} \\ \text { minus } & -x_{1} & y_{1} & z_{1} & x_{2} & y_{2} & z_{2} & x_{3} & y_{3} & z_{3} \\ \text { plus } & -x_{1} & y_{1} & z_{1} & x_{2}-y_{2} & z_{2} & x_{3} & y_{3} & z_{3} \\ \text { minus } & x_{1} & y_{1} & z_{1} & x_{2} & -y_{2} & z_{2} & x_{3} & y_{3} & z_{3} .\end{array}$

The use of parallel or RC dynamics or a mixture will again produce a stable and correct overlap with a suitable test function not orthogonal to $\psi_{A}(R)$.

As with the two-dimensional case, one may use pairs of walkers if one chooses horizontal or vertical pairs according to which is closer.

It is clear that a four body harmonic oscillator can also be treated in the same way. An unpolarized state is an immediate generalization of the discussion for p-states given above. A fully polarized state can be treated by the next step in the development given just above for Eq. 6.5. Since the Slater determinant will now be trilinear, with terms like $x_{1} y_{2} z_{3}$, the elementary ensemble is an octet. We have not experimented with this system, but its stability seems assured. As with previous systems, it also seems very likely that stability will be retained with the use of pairs alone, when appropriate switching among orientation of pairs is arranged.

\section{CONCLUSIONS AND PROSPECTS}

We believe that the work described here represents a genuine advance in our understanding of the structure of algorithms for fermion Monte Carlo. It is true, of course, that harmonic oscillator systems are rather special, and that we have exploited aspects of their particular structure. From an analytical point of view, the fact that excited states are generated by differentiation is a guide to the selection of useful geometrical shapes of correlated ensembles. From the perspective of quantum Monte Carlo, the choice of the ground state 
as an importance function implies a special character of the "drift" that permits the shapes to be preserved. We can then devise patterns of correlated diffusion that also preserve the shapes. The combination breaks the plus-minus symmetry in a special way.

Used in a straightforward way, these methods lead to ensembles that grow exponentially with excitation, but we have seen that in particular examples, pairs of walkers are sufficient. This remains to be demonstrated more generally, and it also remains to be shown that the use of pairs alone gives a method whose computational complexity grows slowly. That is, it needs to be shown that that the logical work of choosing the right orientation for pairs grows slowly, and that the variance does not grow rapidly, when pairs are used.

The most serious work remains, namely, of translating these ideas into equivalent or alternative methods that apply to systems of physical interest. For few-body systems, e.g. few-electron atoms, the point of view developed here is relevant: the triplet state of atomic helium is analogous to the excited s-state of the harmonic oscillator discussed here, and the ground state of the Be atom can be built from two such states. It is likely that the general point of view will also hold: geometrical structures that can be made stable with respect to correlated stochastic dynamics will permit stable fermion Monte Carlo. We plan to pursue these and additional analogs in future research. There are additional kinds of correlated dynamics, not discussed in this paper, that can be applied in new problems.

\section{ACKNOWLEDGMENTS}

We are most grateful to the FORUM of the Istituto Nazionale Fisica della Materia for its support of our visit to the Scuola Normale Superiore in Pisa where much of this paper was written. That visit provided a welcome opportunity to reflect upon the work described here and to bring it to a more coherent state. Many thanks are owing to Luciano Reatto, Stefano Fantoni for their invitation, and to Mario Tosi for the hospitality of the SNS.

We are pleased to acknowledge helpful conversations with Luciano Reatto, Stefano Fantoni, and with Geoffrey Chester, Zhiping Liu, Shiwei Zhang, and Cyrus Umrigar.

This research was conducted using the resources of the Cornell Theory Center, which receives major funding from the National Science Foundation, and New York State. Additional funding comes from the Advanced Research Projects Agency, the National Institutes of Health, the IBM Corporation and other members of the Center's Corporate Research

Institute. MHK is supported in part by the NSF under grant No. DMR-9200469. KES is supported by NSF grant CHE-9407309.

One of us (MHK) offers this paper as tribute to Bernard Jancovici in gratitude for twenty-five years of friendship and professional support.

\section{APPENDIX: THE HARMONIC OSCILLATOR IMPORTANCE SAMPLED GREEN'S FUNCTION}

The imaginary time Green's function, $\rho\left(x, x^{\prime}, \tau\right)$, satisfies the Bloch equation,

$$
H \rho=-\frac{\partial \rho}{\partial \tau}
$$


with the initial condition

$$
\rho\left(x, x^{\prime}, 0\right)=\delta\left(x-x^{\prime}\right) .
$$

In units where $\hbar=m=\omega=1$, the Hamiltonian is

$$
H=-\frac{1}{2} \frac{\partial^{2}}{\partial x}+\frac{1}{2} x^{2}
$$

and the ground state is

$$
\psi_{0}(x)=\frac{1}{\pi^{\frac{1}{4}}} \exp \left(-\frac{x^{2}}{2}\right) .
$$

The exact result $[6]$ is

$$
\rho\left(x, x^{\prime}, \tau\right)=\frac{1}{\sqrt{2 \pi \sinh (\tau)}} \exp \left(-\frac{x^{2}+x^{\prime 2}}{2 \tanh (\tau)}+\frac{x x^{\prime}}{\sinh (\tau)}\right) .
$$

The importance sampled Green's function is

$$
\tilde{G}\left(x, x^{\prime}, \tau\right)=\frac{\psi_{0}(x)}{\psi_{0}\left(x^{\prime}\right)} \exp \left(\frac{\tau}{2}\right) \rho\left(x, x^{\prime}, \tau\right)
$$

where we have included an $\exp (\tau / 2)$ weight factor which corresponds to taking the trial energy $E_{T}$ equal to the ground-state energy $1 / 2$. Combining Eqs. A4-A6, the result for the importance sampled harmonic oscillator Green's function is,

$$
\tilde{G}\left(x, x^{\prime}, \tau\right)=\frac{1}{\sqrt{\pi(1-\exp (-2 \tau))}} \exp \left(-\frac{\left(x-\exp (-\tau) x^{\prime}\right)^{2}}{1-\exp (-2 \tau)}\right)
$$

which is a gaussian centered on $\exp (-\tau) x^{\prime}$ with variance $(1-\exp (-2 \tau)) / 2$. The Ndimensional importance sampled Green's function is a product of one dimensional Green's functions and has the same form. 


\section{REFERENCES}

[1] M. H. Kalos, Phys. Rev. E53, 5420 (1996).

[2] Z. Liu, S. Zhang, and M. H. Kalos, Phys. Rev. E50, 3220 (1994).

[3] P. J. Reynolds, D. M. Ceperley, B. J. Alder, and W. A. Lester, J. Chem Phys. 77, 5593 (1982).

[4] K. E. Schmidt and M. H. Kalos, in Monte Carlo Methods in Statistical Physics II, Topics in Current Physics, edited by K. Binder (Springer-Verlag, Berlin, 1984), p. 125.

[5] D. Arnow, M. H. Kalos, M. A. Lee, and K. E. Schmidt, J. Chem. Phys 77, 5562 (1982).

[6] R. P. Feynman, Statistical Mechanics (Addison-Wesley, Redding, 1972). 